\title{
Mania Episode in a Patient with Phelan Mcdermid Syndrome: A Case Report
}

\author{
Gonzalo Salazar de Pablo* and Julio David Vaquerizo Serrano \\ Department of Defense studies, UK \\ *Corresponding author: Gonzalo Salazar de Pablo, Department of Child and Adolescent Psychiatry, London, UK
}

Keywords: Phelan McDermid; Mania; Bipolar Disorder.

\section{Introduction}

Phelan McDermidsyndromeisageneticand neurodevelopmental condition caused by deletion of chromosome 22q13, by loss of genetic material from the terminal end of chromosome 22 [1]. The vast majority of affected patients present intellectual disability, with an important impact on learning and language [2]. They may also suffer from muscle pathology, like hypotonia and visual defects. Likewise, they suffer neuropsychiatric issues as seizures and behavioural alterations, as well as autism spectrum disorder features [3]. Atypical bipolar disorder has been described in these patients too [3]. Although the deletion can sometimes be detected by high resolution chromosomal analysis, fluorescent in situ hybridization (FISH) or comparative genomic hybridization (CGH) is recommended to confirm the diagnosis $[2,4]$. There is no aetiological treatment for this syndrome. Treatment is based on comorbid pathologies and symptomatic management, although new treatments are being studied [4].

\section{Clinical Case}

A 15-year-old male was brought to the emergency room and later was admitted into the Psychiatry Adolescent Unit due to aggressive behaviour. The patient had been diagnosed with intellectual disability since childhood. Also, language development alterations and features had been detected. After presenting several episodes of psychomotor agitation as well as panic episodes, while he was suffering from several urinary and fungal infections, complete organic screening was performed, including a genetic study. Fluorescent in situ hybridization (FISH) showed a SHANK3 mutation in chromosome 22, a result compatible with Phelan
McDermid syndrome, diagnosis that was established. The patient's phenotype was compatible with this syndrome.

One month later, the patient presented several episodes of anxiety without any trigger. Psychomotor restlessness and escalating physical aggression toward objects at home appeared, while he became increasingly expansive and irritable. Sometimes he presented unmotivated laughter and soliloquies. Sleep disturbances were significant as well, including decreased need for sleep. Progressively greater difficulties to stay calm at home appeared and the patient was admitted into the Psychiatry Unit. After the young person was evaluated and explored, the diagnosis of mania episode was established and the patient was initiated on Quetiapine and Valproic Acid, which lead to a significant improvement.

\section{Conclusion}

a) Studies to detect genetic disorders as Phelan McDermid syndrome should be arranged in patients suffering from severe psychiatric disturbances, especially if they have dysmorphic features.

b) Psychiatric co-morbidity may be present in patients affected with genetic disorders as Phelan McDermid syndrome and this co-morbidity includes severe psychiatric features as mania and psychotic symptoms.

\section{References}

1. Costales J, Kolevzon A (2015) Phelan-McDermid Syndrome and SHANK3: Implications for Treatment. Neurotherapeutics 12(3): 620-630. 
2. Harony-Nicolas H, De Rubeis S, Kolevzon A, Buxbaum J (2015) Phelan McDermid Syndrome: From Genetic Discoveries to Animal Models and Treatment. J Child Neurol 30(14): 1861-1870.

3. Verhoeven W, Egger J, Willemsen M, de Leijer G, Kleefstra T (2012) Phelan-McDermid syndrome in two adult brothers: Atypical bipolar

(C)

To Submit Your Article Click Here: Submit Article

DOI: $10.32474 /$ PRJFGS.2018.02.000150 disorder as its psychopathological phenotype? Neuropsychiatr Dis Treat 8: 175-179.

4. Costales J, Kolevzon A (2015) Phelan-McDermid Syndrome and SHANK3: Implications for Treatment. Neurotherapeutics 12(3): 620-630.

\begin{tabular}{|c|c|c|c|}
\hline & $\begin{array}{c}\text { Peer Reviewed Journal of Forensic } \\
\text { \& Genetic Sciences }\end{array}$ \\
\hline Assets of Publishing with us \\
\hline Global archiving of articles
\end{tabular}

